\title{
Accuracy and Bias in Retrospective Symptom Reporting
}

Omer Van den Bergh \& Marta Walentynowicz Health Psychology, University of Leuven, Belgium

Correspondence to:

Omer Van den Bergh, PhD

Health Psychology

University of Leuven

Tiensestraat 102

B-3000 Leuven

Belgium

Tel: +3216326058

Email: omer.vandenbergh@kuleuven.be 


\section{Purpose of review}

Self-reported bodily symptoms are of primary importance in health care and in health-related research. Typically they are assessed in clinical interviews or by means of traditional questionnaire formats that require the respondent to provide retrospective symptom estimates rated along intuitive frequency and/or intensity standards and aggregated across varying or unspecified time windows.

\section{Recent findings}

Retrospective symptom assessments are often biased when compared to (averaged) momentary assessments of symptoms. A variety of factors and conditions have been identified to influence the amount of bias in symptom reporting. Recent research has focused on the underlying mechanisms for the discrepancy between memory and experience. It is suggested that different types of questions and formats assess different types of information that each may be relevant for other purposes. Knowledge of these underlying mechanisms also provides a relevant framework to better understand individual differences in symptom reporting, including somatoform and somatic symptom disorder.

\section{Summary}

Accuracy of self-reported bodily symptoms is important for the clinician and the researcher. Understanding the mechanisms underlying bias may be provide an interesting window to understand how symptom episodes are processed, encoded and consolidated in memory and provide clues to modify symptom experiences.

\section{Keywords}

Autobiographical memory, episodic memory, somatoform disorder, self-report, symptoms 


\section{Introduction}

Retrospective symptom reports play a primary role when patients consult their physician. They serve as a major source of information for further testing, clinical diagnosis, functional assessment, and informed treatment choice. Also researchers assess self-reported symptoms using standardized questionnaires and rating scales to answer a multitude of health-related research questions. Symptom questionnaires typically require the respondent to provide retrospective symptom estimates rated along intuitive frequency and/or intensity standards (seldom, frequent, almost daily; low, medium, high, etc.) and aggregated across varying or unspecified time windows (e.g. the past week, month, etc.) [1]. Such intuitive integrations of memory-based information rely on complex psychological processes that are often biasing selfreports, that is, they may not correspond closely with actually experienced symptoms.

Biases in self-reports have contributed to concerns about the validity of traditional questionnaires and to the increasing use of momentary ambulatory assessments in real time and context (ecological momentary assessment, EMA) in order to obtain more accurate, experience-near data [e.g., 2]. Since the latter are considered to provide a more objective standard, much research has been devoted to establish the amount of and the conditions for bias in retrospective symptom reports by comparing them to momentary assessments. Recently, however, research shifted towards understanding the processes underlying answers to questions about one's internal state [3]. This perspective has also opened a way to improve our understanding of stable individual differences that are reflected in elevated symptom reports, such as observed in persons with high habitual levels of symptoms, and patients with socalled medically unexplained symptoms, and somatoform and functional somatic 
disorders. In this review, we will address these issues with a focus on bodily symptoms, and occasionally extend our discussion to affective and mood-related symptoms.

\section{Accuracy and bias in retrospective symptom reports}

Symptom questionnaires are often found to overestimate actually experienced symptom levels as indicated by a discrepancy between recalled symptoms and averaged EMA. This so-called memory-experience gap [4] is found in children $\left[5,6^{*}\right.$, $7-9]$ as well as in adult patients $[10-14]$ and healthy persons $\left[15,16,17^{* *}, 18^{* *}\right]$. Also emotions and positive and negative affect are often retrospectively overreported $\left[7,19,20,21^{*}\right]$ which may contribute to an illusion of positive change when comparing retrospective measures of pre-therapeutic distress with actual assessment of distress [22]. However, also accurate retrospective memory as well as underestimation of symptoms have occasionally been reported $\left[23-26,27^{*}\right]$. Several factors appear to influence bias in symptom reports (see Table 1).

Insert Table 1 here

EMA provide in general more accurate and rich information by allowing to assess the variability and context dependency of symptoms. However, the burden on the respondent is rather high and sometimes application of EMA is not feasible. Alternative methods in-between EMA and classic questionnaires have been developed to assess somatic and affective symptoms in everyday life such as end-ofday diaries and the Day Reconstruction Method [53-55]. These methods may also 
provide reliable estimates of symptom intensity levels as well their variability, especially if collected multiple times across the reporting period $[56,57]$.

\section{Understanding bias}

As symptom episodes are experienced, they are processed, encoded and consolidated by the individual in functional memory (sub)systems that contribute to self-referential thinking about one's present, past and future, and help to maintain self-identity. One functional subsystem is autobiographical memory [or self-memory system; 58,59] which in the case of symptom episodes, represents unique healthrelated information that is contextualized in time and space and has particular relevance for the self. Another functional subsystem is personal semantic memory $\left[60,61^{*}\right]$ that represents information about the self that is abstracted from experience and devoid of unique contextual details, implying beliefs about one's personality, roles and general autobiographical facts. Symptom schemata and illness beliefs, reflecting commonalities across multiple symptom and illness episodes as well as semantic knowledge and lay beliefs, can be considered part of personal semantic memory. These theoretical concepts can help to understand bias in retrospective symptom reporting [see Conner \& Feldman Barrett, 3].

Because the memory-experience gap, e.g. retrospective overestimation of symptoms as compared to averaged momentary assessments, appears immediately after a symptom episode $\left[17^{* *}, 35\right]$ and remains rather stable across subsequent measurements over a two-week period $\left[17^{\star *}\right]$, it is unlikely to simply result from memory decay over time. Such findings are more compatible with an idea that different types of measurement gauge different types of information, and possibly assess different "functional selves" [3,62]. First, momentary assessments reveal experiential knowledge or information provided by an experiencing self in a "here- 
and-now" context. They are therefore more closely related than retrospective measures to objective measures of biobehavioral processes involved in being and behaving in that context, such as parameters of autonomic, neuro-endocrine and immune functioning as well as to affective networks in the brain that are associated with experiencing a particular state [63-65].

Second, retrospective assessments about a proximal past may probe information as it has been encoded and consolidated in autobiographic episodic memory. It reflects information from a "remembering self" that may have more interest in being adaptive to guide future behaviour rather than being accurate in representing the past [59]. For example, it may be more relevant to remember how unpleasant an aversive bodily experience was at its peak and how much it got better at the end rather than to remember (and equally weighing) every single moment of the experience. In other words, the peak-end bias in retrospective symptom reports may represent an adaptive bias $\left[17^{* *}, 33,37\right]$. Interestingly, the peak-end rule predicted recalled labor pain up to 2 months after delivery confirming that labor duration is largely neglected. However, this effect was diluted in multiparous compared to primiparous mothers, showing that previous experience moderates the peak-end memory rule [36].

Third, probing information of a more distal past may address personal semantic memory rather than integrated and remembered episodes, reflecting a "believing self" that is more influenced by beliefs about oneself as an historical person with a particular personality, gender and cultural context. Obviously, both episodic and semantic systems may be involved in retrospective ratings to different degrees depending on factors, such as the retention interval and the availability of details [66]. 
Recent findings suggest that when retention intervals extend 3-7 weeks also semantic elements are used to reconstruct hedonic evaluations of past episodes [67].

The view that different ways of assessment tap into different sources of information has important consequences. When the relationship between self-reports and objective physiological measures is the focus of interest, EMA will generally result in more valid data. However, when the goal is to understand and predict future health-related decisions, treatment adherence and illness behavior of patients, remembered symptoms, symptom schemata and illness beliefs may be more relevant than actually experienced symptoms as assessed by EMA [3,68$\left.70,71^{*}, 72^{*}\right]$

\section{Understanding individual differences in symptom overreporting}

The experience of a symptom entails a sensory-perceptual component referring to intensity, location, and other qualities, and an affective-motivational component providing the drive for action to preserve the integrity of the body [73-77]. A question prompting a symptom report from the individual requires an intuitive integration of both components into one global symptom report. This process is influenced by emotional states [76]. So, it is no surprise that symptom reports tend to be more elevated with higher state anxiety, distress, as well as in individuals scoring high for trait negative affectivity, a personality trait characterized by an overreactive evaluative system, elevated threat sensitivity, and vulnerability to negative emotions (see Table 1). A positive relationship between trait negative affectivity and symptom reporting shows up during controlled symptom inductions [78-81] as well as in the absence of physiological dysfunction [81-83]. High trait negative affectivity also characterizes high habitual symptom reporters among healthy persons $\left[84^{*}, 85\right]$ as 
well as patients with somatoform disorder $[86,87]$. Interestingly, although the anxiety and depressive component of negative affectivity are highly correlated, the first appears more associated with concurrent symptom overreporting, whereas the latter with retrospective overreporting [49]. Studies further show that anxious states and appraisals during symptom episodes mediate the effect of trait negative affectivity on symptom overreporting $\left[7,17^{\star *}\right.$; see Table 1$]$, whereas reappraisal during the experience or a focus on sensory rather than on affective aspects of the experience counteracts overreporting in anxious persons $\left[18^{* *}, 88\right]$.

An interesting new hypothesis is that enhanced affective-motivational responding to threatening somatic events may go at the expense of detailed sensory-perceptual processing, blurring a clear distinction between an emotional and a somatic state. This may explain a number of recent findings. First, high habitual symptom reporters and patients with somatoform disorders show diminished correspondence between induced physiological reactions and self-reported symptoms $[81,89]$. Second, elevated symptom reports can be elicited in these persons by simply inducing negative affect (through picture viewing) despite the absence of differences in physiological arousal $\left[83,84^{*}, 90,91^{*}\right]$, suggesting that presenting affective cues substantially biases the experience of a somatic state. Third, patients with somatoform disorder do not exhibit a peak-end memory bias after an experimentally induced dyspnea episode $[37,88]$ : Despite an identical physiological response pattern compared to healthy controls, exhibiting a clear peak and an end with lower intensity, patients' retrospective integration of the symptom episode was not affected by the actual changes in symptoms over the period. Fourth, when given health-related cue words for autobiographical memory retrieval, patients with somatoform disorder exhibit reduced autobiographical memory specificity after controlling for depression 
and rumination $\left[92^{* *}\right.$. Also this finding suggests that somatoform patients process and encode health-related episodes in memory in a little detailed way. Fifth, retrospectively reported symptom intensity of a distressing somatic experience increases over a two-week period in high habitual symptom reporters $\left[17^{* *}\right]$. Interestingly, by manipulating the processing focus towards affective aspects while experiencing a symptom episode this effect is reproduced regardless of pre-exisiting individual differences, whereas focusing on sensory-perceptual aspects eliminates this effect [88]. These results consistently suggest that understanding elevated symptom reporting in high habitual symptom reporters and in patients with somatoform disorder may shed light on the specific way they have experienced and encoded symptom episodes and retrieve them from memory when reporting symptoms in response to questions and questionnaires.

\section{Implications for future research}

Research conducted in the past two decades has considerably changed our view on assessment of bodily (and other) symptoms. It became clear that several variables moderate which type of information is reflected in the ratings and that different types of information may be relevant for different types of outcomes. It is obvious that further investigation of the psychological processes by which a response to a question comes about is of paramount importance in two ways: first, to improve measurement procedures and, second, to understand how psychological processes leading to systematic bias in particular patient groups may be intertwined with and inform about pathological processes, and, as such, provide a window for intervention.

As to the first goal, measuring symptoms and more broadly, health and well-being, in an accurate, valid and reliable way, EMA is uniquely indicated to capture levels, 
variability, temporal relationships and context-dependency of self-reported healthrelated variables [e.g., 93]. The increasing availability of wearable technology and body sensors will ever more allow to measure self-reported variables conjointly with (psycho)physiological responses and create unprecedented opportunities to collect data from "experiencing selves" in real time and space. At the same time, these new measurement strategies pose important new theoretical and methodological challenges for the future [94]. But also within the realm of more paper-and-pencil tests, a variety of sophisticated yet practical measures and measurement approaches (e.g. based on item-response theory and computer adapted testing) are increasingly being developed and made available by the Patient-Reported Outcomes Measurement Information System initiative [95].

As to the second goal, the findings above suggesting that an overreactive evaluative system, possibly combined with reduced sensory-perceptual processing, may play a crucial role in systematic symptom overreporting. This may open a window to not only change bias in symptom reporting, but also change the very psycho(patho)logical processes themselves. For example, modulating the affective evaluation of the experience has recently been demonstrated to counteract bias in retrospective self-reports $\left[18^{* *}, 88,96\right]$. Also improving sensory-perceptual processing by means of interoceptive training reduced the levels of symptom reports in somatoform patients [97], while EMA combined with symptom reattributions in real time resulted in reduced symptom reports $\left[98^{*}\right]$.

Another strategy could be used to modify already consolidated memories of health-related events through, for example, a retrieval-induced forgetting (RIF) task. Using this task, Marche, Briere, and von Baeyer [99*] showed that guided recurrent recall of positive details of initial pain memory in children led to forgetting of negative 
aspects which subsequently affected coping strategies. Reactivating autobiographical and emotional memories of symptom episodes and subsequently updating them via a process of reconsolidation [e.g., 100,101**] could be a useful strategy to modify somatic memories in patients with somatoform and somatic symptom disorder, which may both help the patient and reduce bias in symptom reporting.

\section{Conclusion}

Clinicians and researchers often rely on retrospective symptom reports in clinical interviews and in traditional questionnaires. However, characteristics of the somatic experience, the retention interval as well as state and trait-related individual differences may influence and bias these reports. Recent research suggests that questions prompting the respondent to provide symptom reports may probe different types of information depending on the recall period. Each type of information is predictive of different outcomes. Understanding stable individual differences in symptom overreporting in somatoform disorders or somatic symptom disorders may also provide a window into pathological processes involved in experiencing and encoding somatic experiences that can be targeted for intervention. 


\section{Key points}

- Retrospective symptom reports in clinical interviews and in traditional questionnaires are often biased.

- Characteristics of the somatic experience, the retention interval, and state and trait-related individual differences are important biasing factors.

- Questions prompting the respondent to provide symptom reports may probe different types of information depending on the recall period. Each type of information is predictive of different outcomes.

- Stable individual differences in symptom overreporting in somatoform disorders or somatic symptom disorders are related to psycho(patho)logical processes involved in experiencing and encoding somatic experiences that can be targeted for intervention. 


\section{Acknowledgements}

The study was funded by Grant OT/10/027 and Grant PF/10/005 from the University Research Council of University of Leuven. 


\section{References}

1. Zijlema WL, Stolk RP, Löwe B, et al. How to assess common somatic symptoms in large-scale studies: a systematic review of questionnaires. J Psychosom Res 2013; 74:459-468.

2. Smyth JM, Stone AA. Ecological momentary assessment research in behavioral medicine. J Happiness Stud 2003; 4:35-52.

3. Conner T, Feldman Barrett L. Trends in ambulatory self-report: the role of momentary experience in psychosomatic medicine. Psychosom Med 2012; 74:327-337.

4. Miron-Shatz T, Stone AA, Kahneman D. Memories of yesterday's emotions: does the valence of experience affect the memory-experience gap? Emotion 2009; 9:885-891.

5. Van Den Brink M, Bandell-Hoekstra ENG, Huijer Abu-Saad H. The occurrence of recall bias in pediatric headache: a comparison of questionnaire and diary data. Headache $2001 ; 41: 11-20$.

*6. Noel M, Rabbitts JA, Tai GG, et al. Remembering pain after surgery: a longitudinal examination of the role of pain catastrophizing in children's and parents' recall. Pain 2015; 156:800-808.

This longitudinal study demonstrates that children's pain memories are affected not only by their own dispositional pain catastrophizing, but even to a greater extent by the catastrophic thinking of a parent.

7. Noel M, Chambers CT, McGrath PJ, et al. The role of state anxiety in children's memories for pain. J Pediatr Psychol 2012; 37:567-579.

8. Rocha EM, Marche TA, von Baeyer CL. Anxiety influences children's memory for procedural pain. Pain Res Manag 2009; 14:233-237. 
9. Chen E, Zeltzer LK, Craske MG, et al. Children's memories for painful cancer treatment procedures: implications for distress. Child Dev 2000; 71:933-947.

10. Broderick JE, Schwartz JE, Vikingstad G, et al. The accuracy of pain and fatigue items across different reporting periods. Pain 2008; 139:146-157.

11. Eich E, Reeves JL, Jaeger B, et al. Memory for pain: relation between past and present pain intensity. Pain 1985; 23:375-380.

12. Giske L, Sandvik L, Røe C. Comparison of daily and weekly retrospectively reported pain intensity in patients with localized and generalized musculoskeletal pain. Eur J Pain 2010; 14:959-965.

13. Stone AA, Broderick JE, Shiffman SS, et al. Understanding recall of weekly pain from a momentary assessment perspective: absolute agreement, between- and within-person consistency, and judged change in weekly pain. Pain 2004; 107:61-69.

14. Friedberg F, Sohl S. Memory for fatigue in chronic fatigue syndrome: the relation between weekly recall and momentary ratings. Int J Behav Med 2008; 15:29-33.

15. Cohen LL, Blount RL, Cohen RJ, et al. Children's expectations and memories of acute distress: short- and long-term efficacy of pain management interventions. J Pediatr Psychol 2001; 26:367-374.

16. Gedney JJ, Logan H. Pain related recall predicts future pain report. Pain 2006; 121:69-76.

**17. Walentynowicz M, Bogaerts K, Van Diest I, et al. Was it so bad? The role of retrospective memory in symptom reporting. Heal Psychol 2015; 34:11661174

This experimental study shows that bias in self-reported dyspnea and pain not only 
appears immediately following the experience but also increases over time for distressing dyspnea. It also highlights the role of affective processes in symptom overreporting.

**18. Hovasapian A, Levine LJ. Reappraisal mitigates overestimation of remembered pain in anxious individuals. Cogn Emot 2016; 30:1 1222 -1231.

This experimental study demonstrates that reappraisal of a painful experience during encoding reduces the recall bias among individuals high in trait anxiety sensitivity. This shows the importance of affective evaluations in retrospective symptom overreporting.

19. Levine LJ, Lench HC, Safer MA. Functions of remembering and misremembering emotion. Appl Cogn Psychol 2009; 23:1059-1075.

20. Thomas DL, Diener E. Memory accuracy in the recall of emotions. J Pers Soc Psychol 1990; 59:291-297.

*21. Bąbel P, Pieniążek L, Zarotyński D. The effect of the type of pain on the accuracy of memory of pain and affect. Eur J Pain 2015; 19:358-368.

This longitudinal study demonstrates that memory for pain and affect associated with medical procedures is influenced by the affective evaluation and the meaning of an experience.

22. Safer MA, Keuler DJ. Individual differences in misremembering prepsychotherapy distress: personality and memory distortion. Emotion 2002; 2:162-178.

23. Jamison RN, Raymond SA, Slawsby EA, et al. Pain assessment in patients with low back pain: comparison of weekly recall and momentary electronic data. J Pain 2006; 7:192-199.

24. Bąbel P. Memory of pain induced by physical exercise. Memory 2016; 24:548- 
559.

This longitudinal study demonstrates that pain induced by running a marathon is retrospectively underestimated and that memory for pain is predicted by experienced initial pain and negative affect.

25. Brodie EE, Niven CA. Remembering an everyday pain: the role of knowledge and experience in the recall of the quality of dysmenorrhoea. Pain 2000; 84:89-94.

26. Phillips KM, Faul LA, Small BJ, et al. Comparing the retrospective reports of fatigue using the fatigue symptom index with daily diary ratings in women receiving chemotherapy for gynecologic cancer. J Pain Symptom Manage 2013; 46:282-288.

*27. Self MM, Williams AE, Czyzewski DI, et al. Agreement between prospective diary data and retrospective questionnaire report of abdominal pain and stooling symptoms in children with irritable bowel syndrome. Neurogastroenterol Motil 2015; 27:1110-1119.

This study shows that under certain conditions concordance between retrospective and concurrent ratings of pain provided by children with IBS can be observed, and advocates for the use of diary methods when possible.

28. Stone AA, Schwartz JE, Broderick JE, et al. Variability of momentary pain predicts recall of weekly pain: a consequence of the peak (or salience) memory heuristic. Pers Soc Psychol Bull 2005; 31:1340-1346.

29. Kikuchi H, Yoshiuchi K, Miyasaka N, et al. Reliability of recalled self-report on headache intensity: investigation using ecological momentary assessment technique. Cephalalgia 2006; 26:1335-1343.

30. Sohl SJ, Friedberg F. Memory for fatigue in chronic fatigue syndrome: 
relationships to fatigue variability, catastrophizing, and negative affect. Behav Med 2008; 34:29-38.

31. Jamison RN, Sbrocco T, Parris WC V. The influence of physical and psychosocial factors on accuracy of memory for pain in chronic pain patients. Pain 1989; 37:289-294.

32. Linton SJ, Melin L. The accuracy of remembering chronic pain. Pain 1982; $13: 281-285$.

33. Kahneman D, Fredrickson BL, Schreiber CA, et al. When more pain is preferred to less: adding a better end. Psychol Sci 1993; 4:401-405.

34. Redelmeier DA, Katz J, Kahneman D. Memories of colonoscopy: a randomized trial. Pain 2003; 104:187-194.

35. Redelmeier DA, Kahneman D. Patients' memories of painful medical treatments: real-time and retrospective evaluations of two minimally invasive procedures. Pain 1996; 66:3-8.

36. Chajut E, Caspi A, Chen R, et al. In pain thou shalt bring forth children: the peak-and-end rule in recall of labor pain. Psychol Sci 2014; 25:2266-2271.

37. Bogaerts K, Wan L, Diest I Van, et al. Peak-end memory bias in laboratoryinduced dyspnea. Psychosom Med 2012; 74:974-981.

38. Feine JS, Lavigne GJ, Thuan Dao T, et al. Memories of chronic pain and perceptions of relief. Pain 1998; 77:137-141.

39. Lefebvre JC, Keefe FJ. Memory for pain: the relationship of pain catastrophizing to the recall of daily rheumatoid arthritis pain. Clin J Pain 2002; 18:56-63.

40. Smith WB, Safer MA. Effects of present pain level on recall of chronic pain and medication use. Pain 1993; 55:355-361. 
41. Meek PM, Lareau SC, Anderson D. Memory for symptoms in COPD patients: how accurate are their reports? Eur Respir J 2001; 18:474-481.

42. Houtveen JH, Oei NYL. Recall bias in reporting medically unexplained symptoms comes from semantic memory. J Psychosom Res 2007; 62:277282.

43. Gedney JJ, Logan H, Baron RS. Predictors of short-term and long-term memory of sensory and affective dimensions of pain. J Pain $2003 ; 4: 47-55$.

44. Everts $B$, Karlson $B$, Währborg $P$, et al. Pain recollection after chest pain of cardiac origin. Cardiology 1999; 92:115-120.

45. Gedney JJ, Logan H. Memory for stress-associated acute pain. J Pain 2004; $5: 83-91$.

*46. Bąbel P. Memory of pain and affect associated with migraine and non-migraine headaches. Memory 2015; 23:864-875.

A longitudinal study showing that distorted memories of affect associated with somatic experience can influence the recall of pain memories.

47. Cutler SE, Larson RJ, Bunce SC. Ropressive coping style and the experience and recall of emotion: a naturalistic study of daily affect. J Pers 1996; $64: 379$ 405.

48. Kent G. Memory of dental pain. Pain 1985; 21:187-194.

49. Suls J, Howren MB. Understanding the physical-symptom experience: the distinctive contributions of anxiety and depression. Curr Dir Psychol Sci 2012; 21:129-134.

50. Larsen RJ. Neuroticism and selective encoding and recall of symptoms: evidence from a combined concurrent-retrospective study. J Pers Soc Psychol 1992; 62:480-488. 
51. Feldman Barrett $L$. The relationships among momentary emotion experiences, personality descriptions, and retrospective ratings of emotion. Pers Soc Psychol Bull 1997; 23:1-14.

52. Safer MA, Levine LJ, Drapalski AL. Distortion in memory for emotions: the contributions of personality and post-event knowledge. Personal Soc Psychol Bull 2002; 28:1495-1507.

53. Krueger AB, Stone AA. Assessment of pain: a community-based diary survey in the usa. Lancet 2008; 371:1519-1525.

54. Kahneman D, Krueger AB, Schkade DA, et al. A survey method for characterizing daily life experience: the day reconstruction method. Science 2004; 306:1776-1780.

55. Broderick JE, Schwartz JE, Schneider S, et al. Can end-of-day reports replace momentary assessment of pain and fatigue? J Pain 2009; 10:274-281.

56. Stone AA, Schneider S, Broderick JE, et al. Single-day pain assessments as clinical outcomes: not so fast. Clin J Pain 2014; 30:739-743.

57. Dockray S, Grant N, Stone AA, et al. A comparison of affect ratings obtained with ecological momentary assessment and the day reconstruction method. Soc Indic Res 2010; 99:269-283.

58. Conway MA. Memory and the self. J Mem Lang 2005; 53:594-628.

59. Conway MA, Pleydell-Pearce CW. The construction of autobiographical memories in the self-memory system. Psychol Rev 2000; 107:261-288.

60. Renoult L, Davidson PSR, Palombo DJ, et al. Personal semantics: at the crossroads of semantic and episodic memory. Trends Cogn Sci 2012; 16:550 558.

${ }^{*} 61$. Renoult L, Tanguay A, Beaudry M, et al. Personal semantics: is it distinct from 
episodic and semantic memory? An electrophysiological study of memory for autobiographical facts and repeated events in honor of Shlomo Bentin. Neuropsychologia 2016; 83:242-256.

This ERP study is the first to show that neural correlates of personal semantics can be differentiated from semantic and episodic memories. Personal semantics, encompassing autobiographical facts and repeated events, seem to be an intermediate form of declarative memory.

62. Kahneman D, Riis J. Living, and thinking about it: Two perspectives on life. In: The science of well-being. Edited by Huppert F, Baylis N, Keverne B (editors). Oxford: Oxford University Press; 2005, pp. 285-304.

63. Kamarck TW, Schwartz JE, Shiffman S, et al. Psychosociol stress and cardiovascular risk: what is the role of daily experience? J Pers $2005 ; 73: 1749$ 1774.

64. Steptoe A, Leigh Gibson E, Hamer M, et al. Neuroendocrine and cardiovascular correlates of positive affect measured by ecological momentary assessment and by questionnaire. Psychoneuroendocrinology $2007 ; 32: 56-64$.

65. Nater UM, Jones JF, Lin J-MS, et al. Personality features and personality disorders in chronic fatigue syndrome: a population-based study. Psychother Psychosom 2010; 79:312-318.

66. Robinson MD, Clore GL. Belief and feeling: evidence for an accessibility model of emotional self-report. Psychol Bull 2002; 128:934-960.

67. Geng X, Chen Z, Lam W, et al. Hedonic evaluation over short and long retention intervals: the mechanism of the peak-end rule. J Behav Decis Mak 2013; 26:225-236.

68. Petrie KJ, Weinman J. Patients' perceptions of their illness: the dynamo of 
volition in health care. Curr Dir Psychol Sci 2012; 21:60-5.

69. Horne R, Chapman SCE, Parham R, et al. Understanding patients' adherencerelated beliefs about medicines prescribed for long-term conditions: a metaanalytic review of the necessity-concerns framework. PLoS One 2013; 8.

70. Noel M, Chambers CT, McGrath PJ, et al. The influence of children's pain memories on subsequent pain experience. Pain 2012; 153:1563-1572.

*71. Chapman SCE, Horne R, Eade R, et al. Applying a perceptions and practicalities approach to understanding nonadherence to antiepileptic drugs. Epilepsia 2015; 56:1398-1407.

This cross-sectional study highlights the importance of patients' perceptions and beliefs in adherence to prescribed medication, also suggesting which modifiable factors could enhance adherence.

*72. Faasse K, Grey A, Horne R, et al. High perceived sensitivity to medicines is associated with higher medical care utilisation, increased symptom reporting and greater information-seeking about medication. Pharmacoepidemiol Drug Saf 2015; 24:592-599.

A study showing that perceived sensitivity to medications in not only prevalent in society, but also related to clinical outcomes such as self-reported somatic symptoms and GP visits, highlighting the role of perceptions and beliefs in symptom reporting.

73. von Leupoldt A, Dahme B. Differentiation between the sensory and affective dimension of dyspnea during resistive load breathing in normal subjects. Chest 2005; 128:3345-3349.

74. von Leupoldt A, Sommer T, Kegat S, et al. The unpleasantness of perceived dyspnea is processed in the anterior insula and amygdala. Am J Respir Crit Care Med 2008; 177:1026-1032. 
75. von Leupoldt A, Sommer T, Kegat S, et al. Dyspnea and pain share emotionrelated brain network. Neuroimage 2009; 48:200-206.

76. von Leupoldt A, Mertz C, Kegat S, et al. The impact of emotions on the sensory and affective dimension of perceived dyspnea. Psychophysiology 2006; 43:382-386.

77. Craig AD. A new view of pain as a homeostatic emotion. Trends Neurosci 2003; 26:303-307.

78. Wan L, Stans L, Bogaerts K, et al. Sensitization in medically unexplained dyspnea: differential effects on intensity and unpleasantness. Chest 2012; 141:989-995.

79. Han J, Zhu Y, Li S, et al. Medically unexplained dyspnea: psychophysiological characteristics and role of breathing therapy. Chin Med J 2004; 117:6-13.

80. Stegen K, Van Diest I, Van de Woestijne KP, et al. Do persons with negative affect have an attentional bias to bodily sensations? Cogn Emot 2001; 15:813829.

81. Bogaerts K, Millen A, Li W, et al. High symptom reporters are less interoceptively accurate in a symptom-related context. J Psychosom Res 2008; 65:417-424.

82. Bogaerts K, Janssens T, De Peuter S, et al. Negative affective pictures can elicit physical symptoms in high habitual symptom reporters. Psychol Health 2010; 25:685-698.

83. Constantinou E, Bogaerts K, Van Diest I, et al. Inducing symptoms in high symptom reporters via emotional pictures: the interactive effects of valence and arousal. J Psychosom Res 2013; 74:191-196.

*84. Bogaerts K, Rayen L, Lavrysen A, et al. Unraveling the relationship between 
trait negative affectivity and habitual symptom reporting. PLoS One 2015; 10:1-15.

This article adds to research on the relationship between negative affectivity (NA) and symptoms by revealing that 1. trait NA is a necessary but not sufficient factor to develop habitual symptom reporting; 2 . symptoms can be induced by simply showing negative affective pictures; 3 . the symptom induction effect is related to difficulty identifying feelings, but not to executive functions.

85. Van Diest I, De Peuter S, Eertmans A, et al. Negative affectivity and enhanced symptom reports: differentiating between symptoms in men and women. Soc Sci Med 2005; 61:1835-1845.

86. de Waal MWM, Arnold IA, Eekhof JAH, et al. Somatoform disorders in general practice: prevalence, functional impairment and comorbidity with anxiety and depressive disorders. Br J Psychiatry 2004; 184:470-476.

87. Henningsen P, Zimmermann T, Sattel H. Medically unexplained physical symptoms, anxiety, and depression. Psychosom Med 2003; 65:528-533.

88. Walentynowicz M. Memory processes in retrospective symptom (over)reporting [dissertation]. Leuven, BE; University of Leuven, 2016.

89. Bogaerts K, Van Eylen L, Li W, et al. Distorted symptom perception in patients with medically unexplained symptoms. J Abnorm Psychol 2010; 119:226-34.

90. Constantinou E, Van Den Houte M, Bogaerts K, et al. Can words heal? Using affect labeling to reduce the effects of unpleasant cues on symptom reporting. Front Psychol 2014; 5:1-11.

*91. Constantinou E, Bogaerts K, Van Oudenhove L, et al. Healing words: using affect labeling to reduce the effects of unpleasant cues on symptom reporting in IBS patients. Int J Behav Med 2015; 22:512-520. 
This experimental study shows that presenting unpleasant cues to patients with IBS leads to increased symptom ratings. This effect can be reduced by emotion regulation strategies.

${ }^{* *} 92$. Walentynowicz M, Raes F, Van Diest I, et al. The specificity of health-related autobiographical memories in patients with somatic symptom disorder. Psychosom Med 2016.

This study reveals a reduced memory specificity among patients with somatic symptom disorder, which suggests that encoding of health-related episodes and related symptoms was less detailed in this disorder.

93. lacob E, Donaldson G, Neikrug A, et al. (121) Self-report ecological momentary assessment in patients with fibromyalgia to examine temporal relationships between pain with mood, fatigue, and sleep. J Pain 2016; 17:S6.

94. Hoppmann CA, Ho A. Ecological Momentary Assessment. In: The Encyclopedia of Adulthood and Aging. Hoboken, NJ, USA: John Wiley \& Sons, Inc.; 2016, p. 1-5.

95. Cella D, Riley W, Stone AA, et al. The patient-reported outcomes measurement information system (PROMIS) developed and tested its first wave of adult self-reported health outcome item banks: 2005-2008. J Clin Epidemiol 2010; 63:1179-1194.

96. Takarangi MKT, Segovia DA, Dawson E, et al. Emotional impact feedback affects how people remember an analogue trauma event. Memory 2014; 22:1041-1051.

97. Schaefer M, Egloff B, Gerlach AL, et al. Improving heartbeat perception in patients with medically unexplained symptoms reduces symptom distress. Biol Psychol 2014; 101:69-76. 
98. Kerstner T, Witthöft M, Mier D, et al. A diary-based modification of symptom attributions in pathological health anxiety: effects on symptom report and cognitive biases. J Consult Clin Psychol 2015; 83:578-589.

This study shows that health anxiety symptoms can be reduced with a diary-based 2week attribution modification training, which could be proposed as an add-on for existing therapies.

*99. Marche TA, Briere JL, von Baeyer CL. Children's forgetting of pain-related memories. J Pediatr Psychol 2016; 41:220-231.

This study demostrates that recalling positive aspects of pain memories can promote forgetting of negative aspects, and suggests that retrieval-induced forgetting task could be used to manage pain memories in childen.

100. Schwabe L, Nader K, Pruessner JC. Reconsolidation of human memory: brain mechanisms and clinical relevance. Biol Psychiatry 2014; 76:274-280.

**101. Lane RD, Ryan L, Nadel L, et al. Memory reconsolidation, emotional arousal, and the process of change in psychotherapy: new insights from brain science. Behav Brain Sci 2015; 38.

An excellent review of the role of emotional arousal and memory reconsolidation in therapeutic change. The article highlights the components essential for the modification of prior memories. 
Table 1. Factors affecting accuracy and bias of retrospective symptom reports.

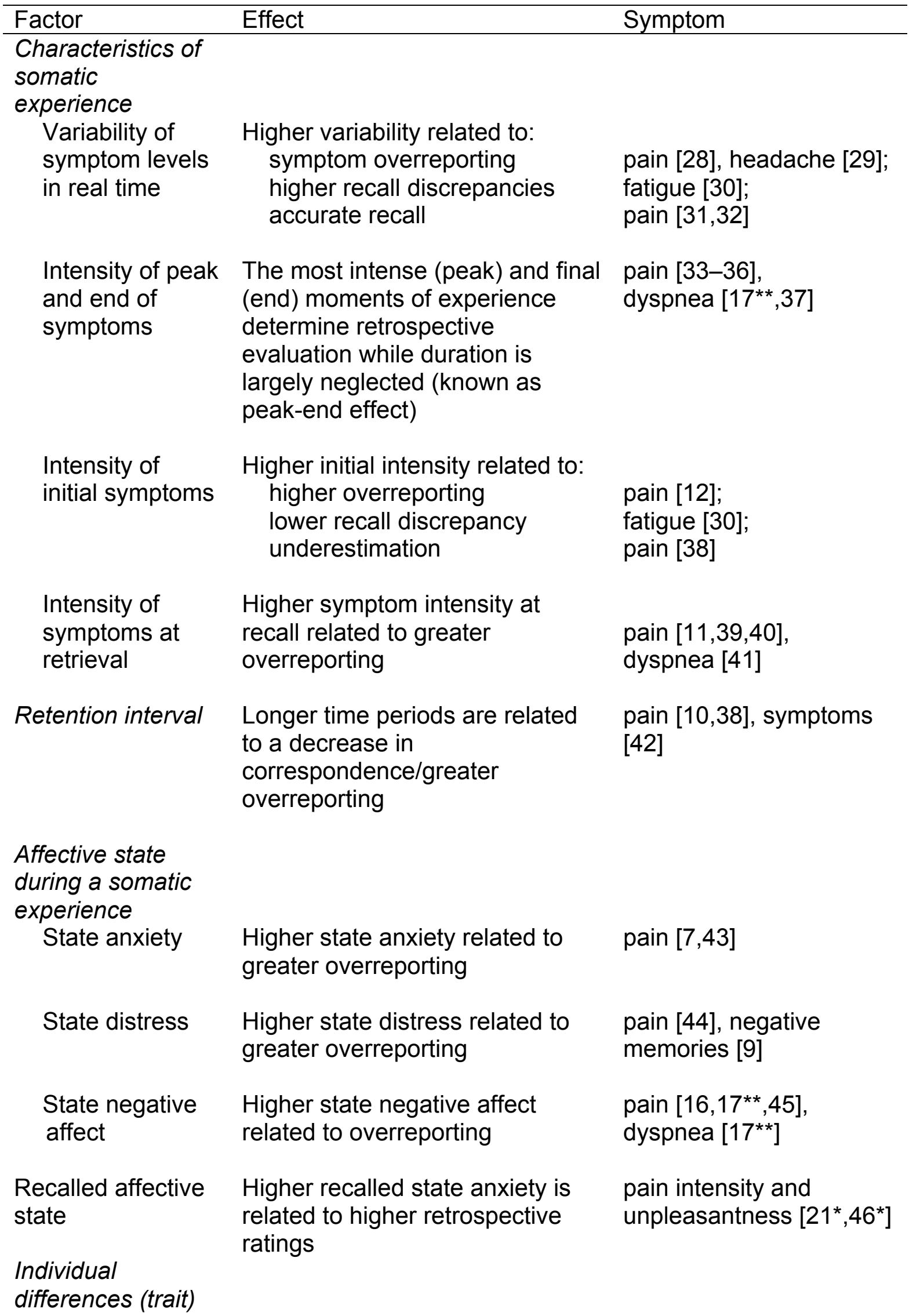


Anxiety

Anxiety sensitivity

Negative affectivity

Depression

\section{Catastrophizing}

H

Higher anxiety sensitivity related to greater overreporting

Higher negative affectivity related to:

greater overreporting

underestimation

Higher depression related to: greater overreporting

lower recall discrepancy

Higher catastrophizing related to:

lower recall discrepancy

higher retrospective ratings

fatigue [30], pain [39];

pain $\left[6^{*}\right]$

Self-esteem, Higher self-esteem and optimism optimism

negative/unpleasant affect [47], pain [8,48], pain-related fear [7], symptoms [49]

pain $\left[18^{\star *}\right]$, pain-related fear [7]

symptoms [50], negative affective states [51,52], pre-treatment distress [22];

positive emotions [52]

negative emotions [52], symptoms [49];

fatigue [30] related to: 